ОРИГИНАЛНИ СТАТИИ

ORIGINAL ARTICLES

\title{
PREVALENCE OF SARCOPENIA IN ADULT PATIENTS WITH ANKYLOSING SPONDYLITIS
}

\author{
M. M. Younis' ${ }^{1}$ K. Z. Mayouf Albedri² \\ ${ }^{1}$ Rheumatology and Rehabilitation Department, Al-Yarmook teaching Hospital, Baghdad Alkarkh Health Directorate, \\ Ministry of Health/Environment - Baghdad, Iraq \\ ${ }^{2}$ Consultant Internal Medicine and Rheumatology, Rheum. and Med. Rehabilitation, Iraqi Board for Medical Specializations, \\ Ministry of higher education and scientific research - Baghdad, Iraq
}

\begin{abstract}
Ankylosing spondylitis is a chronic, progressive inflammatory rheumatic disease that involves primarily the sacroiliac joints and the axial skeleton. Sarcopenia is characterized by progressive and generalized loss of skeletal muscle mass and strength with a risk of adverse outcomes such as physical disability and poor quality of life. The study aimed to assess the prevalence of sarcopenia in patients with ankylosing spondylitis. A cross-sectional study was conducted at Rheumatology Unit from January 2019 to July 2019. The study population consisted of 50 Iraqi patients diagnosed with AS. Demographic data, physical activity using General Practice Physical Activity Questionnaire (GPPAQ), disease activity scores using Bath Ankylosing Spondylitis Disease Activity Index (BASDAI) were recorded. Sarcopenia assessment was done by using a dual-energy $x$-ray absorptiometry scan for body composition analysis and measuring skeletal mass index (SMI). The maximal voluntary grip strength of the hand was measured with an electronic dynamometer. Physical function was assessed by a 4-meter usual gait speed test. The prevalence of presarcopenia was $6 \%$, while sarcopenia was documented in $10 \%$ of patients. The mean value of body mass index (BMI) is significantly lower in patients with sarcopenia $(p=0.001)$. The vast majority of the sarcopenic group $(80 \%)$ were physically inactive which was statistically significant compared with the non-sarcopenic group $(p=0.033)$. No significant association of treatment with anti-TNF or its duration was found between sarcopenia and non-sarcopenia groups ( $p=0.377 ; p=0.187$ ). Both lean mass $(L M)$ and handgrip showed fair validity to differentiate between AS patients. Patients with AS are at higher risk of developing early sarcopenia. Lower BMI and longer disease duration increase the risk of sarcopenia, while differences in gender, smoking and the use of anti-TNF do not influence the risk. Physical activity may improve muscle strength and perhaps decrease the risk.
\end{abstract}

Key words: ankylosing spondylitis, sarcopenia, presarcopenia, anti-TNF

\section{INTRODUCTION}

Ankylosing Spondylitis (AS) is a chronic, progressive inflammatory rheumatic disease that involves primarily the sacroiliac joints and the axial skeleton. It is the major subtype and the main outcome of an inter-related group of rheumatic diseases named spondyloarthritides [1, 2]. Age of disease onset usually peaks in the second and third decades of life. Approximately $80 \%$ of patients with AS experience symptoms at $\leq 30$ years of age, while only $5 \%$ will present with symptoms at $\geq 45$ years of age [3]. The estimated prevalence of AS in Iraq was $0.9 \%$ [4]. Low back pain and stiffness is the most common symptom and the first manifestation in approximately $75 \%$ of patients [5].

Sarcopenia is a progressive and generalized skeletal muscle disorder that is associated with an increased likelihood of adverse outcomes including falls, fractures, physical disability, and mortality [6]. The term sarcopenia is derived from the Greek words sarx (flesh) and penia (poverty), describes a condition characterized by loss of muscle mass and muscle strength [7].

Baumgartner et al. used a Skeletal muscle index cutoff of -2SDs below the mean of a young reference group, and the prevalence of sarcopenia ranged from $13 \%$ to $24 \%$ in persons aged 65 to 70 years and 
was over $50 \%$ for those older than 80 years [8]. Another study reported that sarcopenia was prevalent in $10 \%$ of men and $8 \%$ of women and that reduced skeletal muscle was independently associated with functional impairment and disability, particularly in older women [9].

The European Working Group on Sarcopenia in Older People (EWGSOP) updates the clinical algorithm for diagnosis in 2018; sarcopenia is probable when low muscle strength is detected. A sarcopenia diagnosis is confirmed by the presence of low muscle quantity or quality. When low muscle strength, low muscle quantity/quality, and low physical performance are all detected, sarcopenia is considered severe [6]. It is divided into three stages for helpful in light of setting appropriate recovery goals as well as selecting treatments and intervention: pre-sarcopenia stage, sarcopenia stage, and severe sarcopenia [10]. According to EWGSOP, sarcopenia is subcategorized into primary and secondary Sarcopenia [6].

AS patients are at risk of accelerated muscle loss. By leading to reduced muscle power, resistance, and physical activity, the loss of muscle mass contributes to the restriction in functional independence and daily living activities [11]. Up to our knowledge, no previously reported study has examined the prevalence of sarcopenia in the Iraqi population, however, some studies estimated the prevalence of sarcopenia in certain diseases like systemic lupus erythematosus [12].

This study assessed the prevalence of sarcopenia in Iraqi patients with AS and evaluated the impact of sociodemographic and clinical characteristics of AS on sarcopenia.

\section{METHODS}

\section{Study design and setting}

A cross-sectional study was conducted among AS patients who presented consecutively to the Rheumatology Unit of Baghdad Teaching Hospital/ Medical City from the period of January 2019 to July 2019. A total of 50 Iraqi patients with AS after fulfilling modified NEWYORK diagnostic criteria) [13] were enrolled in the study.

\section{Selection criteria}

1. Inclusion criteria were defined as any cooperative individual aged between 18 to 60 years with primary AS regardless of the disease onset.

2. Exclusion criteria were defined as any individual younger than 18 years old, or any individual not keen to participate in the study, any overlapping with another rheumatological disease, pregnancy; possible other causes of secondary sarcopenia (malnutrition, diabetes, chronic kidney disease, chronic obstructive pulmonary disease, heart failure); recent steroid therapy (over the last three months) and presence of metacarpophalangeal joint derangement.

\section{Data collection}

All medical procedures were done after obtaining oral informed consent from the participants. A private subject interview was done including full medical and drug history. Data were collected using a pre-constructed data collection sheet, protocol for each participant (Appendix 1, 2, 3), (Appendix 4 ), and (Appendix 5) $[6,14]$. The physical activity of each participant was assessed by the General Practice Physical Activity Questionnaire (GPPAQ) (Appendix 6) [15].

\section{Measuring sarcopenia parameters}

Body composition measurements: a dual-energy $x$-ray absorptiometry scan (DXA) scan (393 rue Charles Lindbergh, 34130 Mauguio France) was used for the body composition analysis. Fat mass, lean mass, and bone mineral density (BMD) were measured by DXA. Total and regional body composition was evaluated by using a whole-body DXA scan. The muscle mass of the four limbs from the DXA scan summed as appendicular skeletal muscle mass (ASMM) and defined SMI was calculated as ASMM/height2 $\left(\mathrm{kg} / \mathrm{m}^{2}\right)$. Cut off point is $<6.0 \mathrm{~kg} / \mathrm{m}^{2}$ for women and $7.0 \mathrm{~kg} / \mathrm{m}^{2}$ for men [6].

Muscle strength: handgrip strength was measured using an electronic hand dynamometer (EH101 CAMRY, China) [16], the width of the dynamometers grip was individually adjusted to the hand size of the participant. Tests were performed in an upright standing position, arms down by the side, and full elbow extension [17]. Two test trials were performed, both for the dominant and non-dominant hand, the best trial was included in the analysis [18]. The cutoff value for handgrip strength $<27 \mathrm{~kg}$ for men and $<16 \mathrm{~kg}$ for women [6].

Physical performance was assessed by the 4-meter usual walking speed test, with speed measured manually with a stopwatch, a single cut-off speed $\leq$ of $0.8 \mathrm{~m} / \mathrm{s}$ for both genders was considered as impaired physical performance [6]. Gait speed is considered a quick, safe, and highly reliable test for sarcopenia, and it is widely used in practice [19].

Pre-sarcopenia was defined according to Baumgartner's definition by SMI $<7 \mathrm{~kg} / \mathrm{m}^{2}$ for men and $<6$ for women. Sarcopenia was defined by 
the combined presence of the two following criteria according to the EWGSOP: a low muscle mass by whole-body DXA and a low muscle strength assessed by the handgrip. Severe sarcopenia was defined by the combination of the three criteria: low muscle mass by whole-body DXA, low muscle strength, and impaired physical performance by Gait speed test.

\section{Ethical consideration}

Ethical approval was obtained from the Ethical Committee of the University of Baghdad, College of Medicine, Rheumatology and Medical Rehabilitation Unit, and following the Declaration of Helsinki. Signed informed consent was taken from each participant in the study.

\section{Statistical analysis}

Statistical presentation and analysis of the present study were conducted using the mean, standard deviation, and Chi-square for linear correlation coefficient, t-test, and ANOVA by SPSS V20. ROCcurve: - Receiver Operating Characteristic curve analysis. Sensitivity, Specificity, PPV, NPV, and accuracy were calculated (Carter J V, 2016). P < 0.05 was considered significant.

\section{RESULTS}

The mean age of AS patients were $(36.64 \pm 7.684)$ years. The studied group consisted of 47 (94\%) men and $3(6 \%)$ women. Smoking was frequently higher in the AS patients. The physical activity was statistically non-significant. The mean BMI in AS patients was (28.447 \pm 6.287 ), (Table 1). From a total of 50 patients with AS, 31 (62\%) were having active disease and 19 $(38 \%)$ were inactive (Figure 1).

About 12 (24\%) patients were not received anti-TNF treatment, while $38(76 \%)$ patients were kept on anti-TNF. Anti-TNF agents included Etanercept, Infliximab, or Adalimumab (Figure 2).

The data showed that mean of disease duration in AS patients were $(6.92 \pm 4.985)$ years, the mean of BASDAI was $(4.16 \pm 1.714)$, and the mean duration of anti-TNF was $(25.816 \pm 24.655)$ months (Table 2).

The prevalence of presarcopenia among AS group was $6 \%$, while the prevalence of sarcopenia was $10 \%$, with no significant ( $p$ 0.152) (Table 3 ).

The results showed that the mean age of AS patients without sarcopenia was $(37.089 \pm 7.192)$ years, whereas those with sarcopenia was (32.6 \pm 11.48 ) years, with no significant differences $(p=$ 0.219 ). The mean value of BMI is significantly low- er in patients with sarcopenia than in those without sarcopenia $(p=0.001)$. Sarcopenic patients were normal-weight $(80 \%)$ and the remaining $(20 \%)$ were underweight ( $p=<0.001$ ), when compared with the non-sarcopenic group, which showed significantly higher BMI (Table 4).

The vast majority of the sarcopenic group (80\%) were physically inactive, which was statistically significant compared with the non-sarcopenic group ( $p$ $=0.033$ ), (Table 5). Handgrip was significantly higher in physically active groups, whereas LM, SMI, and gait speed were of no significant over subgroups (Table 6).

There was no significant association of treatment with anti-TNF or its duration between sarcopenia and non-sarcopenia groups $(p=0.377$ and $p=$ 0.187), respectively (Table 7).

Longer disease duration is significantly associated with sarcopenia $(p=0.008)$, while disease activity is insignificantly associated with sarcopenia $(p=$ 0.382) (Table 8).

Both LM, and handgrip showed fair validity in AS patients by applying Receiver operating characteristic (ROC), sensitivity (68\%), specificity $(68 \%)$ when the area under the curve (AUC) was 0.71. Both SMI, and usual gait speed failed in the AS patients (AUC) for SMI 0.537, and the usual gait speed test was 0.546 (Table 9) (Figures 3, 4, 5).

Table 1. Demographic and clinical characteristics of AS patients

\begin{tabular}{|c|c|c|c|c|}
\hline \multicolumn{2}{|l|}{ Variable } & \multicolumn{2}{|l|}{ Patients } & \multirow{3}{*}{ **P-value } \\
\hline & & No. & $\%$ & \\
\hline Age (years) & Mean \pm SD & \multicolumn{2}{|c|}{$36.64 \pm 7.684$} & \\
\hline \multirow[t]{2}{*}{ Gender } & Male & 47 & 94 & \multirow{2}{*}{0.05} \\
\hline & Female & 3 & 6 & \\
\hline \multirow{4}{*}{$\begin{array}{l}\text { BMI } \\
\left(\mathrm{kg} / \mathrm{m}^{2}\right)\end{array}$} & Underweight & 1 & 2 & \multirow{4}{*}{0.733} \\
\hline & Normal & 12 & 24 & \\
\hline & Overweight & 20 & 40 & \\
\hline & Obese & 17 & 34 & \\
\hline \multirow{3}{*}{ Smoking } & Active smoking & 17 & 34 & \multirow{3}{*}{0.04} \\
\hline & Non-smoking & 22 & 44 & \\
\hline & Ex-smoking & 11 & 22 & \\
\hline \multirow{4}{*}{$\begin{array}{l}\text { Physical } \\
\text { activity }\end{array}$} & Inactive & 13 & 26 & \multirow{4}{*}{0.94} \\
\hline & \begin{tabular}{|l} 
Moderate \\
Inactive
\end{tabular} & 18 & 36 & \\
\hline & Moderate active & 12 & 24 & \\
\hline & Active & 7 & 14 & \\
\hline
\end{tabular}

BMI, body mass index; SD, standard deviation; ${ }^{* *}$ Chi-square of linear correlation coefficient 


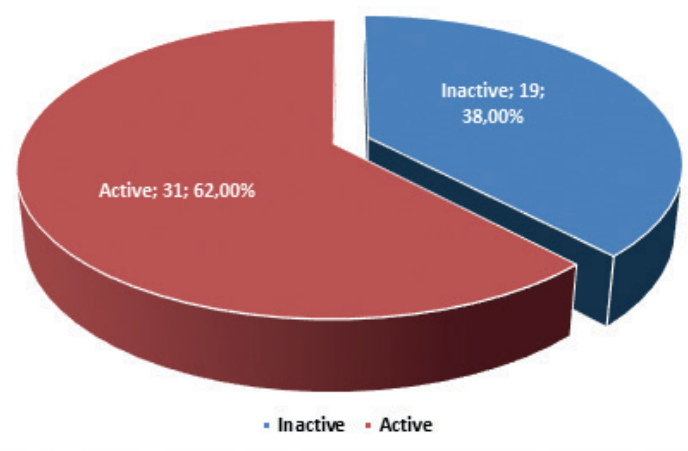

Fig. 1. Disease activity in AS group

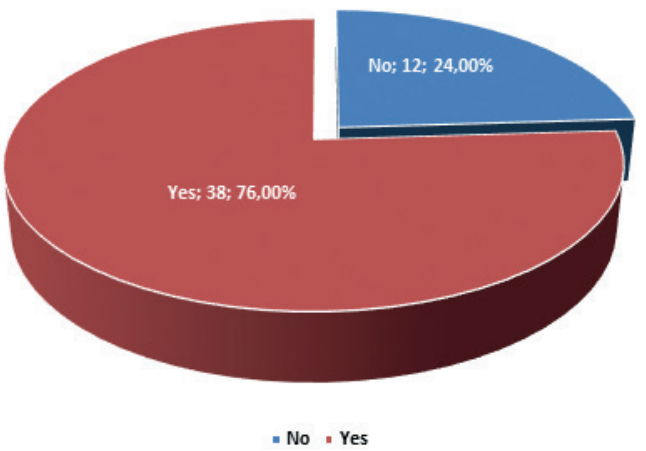

Fig. 2. Anti-TNF treatment in AS group

Table 2. Mean values of disease duration, BASDAl, and duration of anti-TNF

\begin{tabular}{|l|c|}
\hline Disease characteristic & Mean \pm SD \\
\hline Disease duration (years) & $6.92 \pm 4.985$ \\
\hline BASDAI & $4.16 \pm 1.714$ \\
\hline Duration of anti-TNF (months) & $25.816 \pm 24.655$ \\
\hline
\end{tabular}

BASDAI, Bath Ankylosing Spondylitis Disease Activity Index; Anti TNF, anti-tumor necrosis factor

Table 3. The prevalence of presarcopenia, sarcopenia in the studied group

\begin{tabular}{|l|c|c|c|}
\hline Results & $\mathbf{n}$ & $\%$ & \multirow{2}{*}{${ }^{* * P}$-value } \\
\hline No sarcopenia & 42 & 84 & \multirow{2}{*}{0.071} \\
\hline Pre-sarcopenia & 3 & 6 & \multirow{2}{*}{0.07} \\
\hline Sarcopenia & 5 & 10 & \\
\hline Total & 50 & 100 & \\
\hline
\end{tabular}

${ }^{* *}$ Chi-square of linear correlation coefficient

Table 4. Mean age and BMI association between sarcopenic and non-sarcopenic

\begin{tabular}{|c|c|c|c|}
\hline & No sarcopenia & Sarcopenia & \multirow{2}{*}{${ }^{* *}$ P-value } \\
\hline & \multicolumn{2}{|c|}{ Mean \pm SD } & \\
\hline Age (years) & $37.089 \pm 7.192$ & $32.6 \pm 11.48$ & 0.219 \\
\hline BMI (kg/m²) & $29.372 \pm 5.908$ & $20.12 \pm 1.974$ & 0.001 \\
\hline
\end{tabular}

BMI, Body Mass Index; ${ }^{*}$ Chi-square of t-test

Table 5. Demographics association between sarcopenia and non-sarcopenia

\begin{tabular}{|c|c|c|c|c|c|c|}
\hline \multirow{2}{*}{\multicolumn{2}{|c|}{ No. }} & \multicolumn{2}{|c|}{ No sarcopenia } & \multicolumn{2}{|c|}{ Sarcopenia } & \multirow{2}{*}{${ }^{*}$ P-value } \\
\hline & & \multirow{2}{*}{$\begin{array}{l}\% \\
42 \\
\end{array}$} & \multirow{2}{*}{$\begin{array}{l}\text { No. } \\
93.33 \\
\end{array}$} & \multirow{2}{*}{$\begin{array}{l}\% \\
5 \\
\end{array}$} & \multirow[b]{2}{*}{100} & \\
\hline \multirow{2}{*}{ Gender } & Male & & & & & \multirow{2}{*}{0.552} \\
\hline & Female & 3 & 6.67 & 0 & 0 & \\
\hline \multirow{4}{*}{ BMI } & Underweight & 0 & 0 & 1 & 20 & \multirow{4}{*}{$<0.001$} \\
\hline & Normal & 8 & 17.78 & 4 & 80 & \\
\hline & Overweight & 20 & 44.44 & 0 & 0 & \\
\hline & Obese & 17 & 37.78 & 0 & 0 & \\
\hline \multirow{3}{*}{ Smoking } & Active smoking & 14 & 31.11 & 3 & 60 & \multirow{3}{*}{0.309} \\
\hline & Non-smoking & 20 & 44.44 & 2 & 40 & \\
\hline & Ex-smoking & 11 & 24.44 & 0 & 0 & \\
\hline \multirow{4}{*}{$\begin{array}{l}\text { Physical } \\
\text { Activity }\end{array}$} & Inactive & 9 & 20 & 4 & 80 & \multirow{4}{*}{0.033} \\
\hline & Moderate Inactive & 17 & 37.78 & 1 & 20 & \\
\hline & Moderate active & 12 & 26.67 & 0 & 0 & \\
\hline & Active & 7 & 15.56 & 0 & 0 & \\
\hline
\end{tabular}

BMI, body mass index; *ANOVA 
Table 6. Physical activity effect on sarcopenia parameters

\begin{tabular}{|l|c|c|c|c|c|}
\hline \multirow{3}{*}{ Parameter } & \multicolumn{4}{|c|}{ Physical activity } & \multirow{2}{*}{} \\
\cline { 2 - 5 } & Inactive & $\begin{array}{c}\text { Moderate } \\
\text { Inactive }\end{array}$ & $\begin{array}{c}\text { Moderate } \\
\text { active }\end{array}$ & \multirow{2}{*}{ Active } & *P-value \\
\cline { 2 - 6 } & \multicolumn{4}{|c|}{ Mean \pm SD } & \\
\hline LM (kg) & $41.866 \pm 6.51$ & $44.49 \pm 5.221$ & $46.862 \pm 6.384$ & $46.203 \pm 7.392$ & 0.214 \\
\hline SMI & $7.791 \pm 1.024$ & $7.804 \pm 0.875$ & $8.372 \pm 0.972$ & $8.267 \pm 1.082$ & 0.315 \\
\hline Handgrip & $32.615 \pm 7.217$ & $28.611 \pm 7.822$ & $35.592 \pm 6.961$ & $39.557 \pm 8.492$ & 0.01 \\
\hline Usual Gait Speed & $0.892 \pm 0.196$ & $0.973 \pm 0.114$ & $0.888 \pm 0.198$ & $0.991 \pm 0.07$ & 0.279 \\
\hline
\end{tabular}

LM, lean mass; SMI, skeletal muscle mass; SD; standard deviation; *ANOVA

Table 7. Anti-TNF treatment association between groups

\begin{tabular}{|l|c|c|c|c|c|c|}
\hline \multirow{2}{*}{ No. } & \multicolumn{2}{|c|}{ No sarcopenia } & \multicolumn{2}{|c|}{ Sarcopenia } & \multirow{2}{*}{ *P-value } \\
\cline { 3 - 6 } & $\%$ & No. & $\%$ & & \\
\hline \multirow{2}{*}{ Anti-TNF } & No & 10 & 22.22 & 2 & 40 & \multirow{2}{*}{0.377} \\
\cline { 2 - 6 } & Yes & 35 & 77.78 & 3 & 60 & \\
\hline \multicolumn{2}{|l|}{ Duration (Mean \pm SD) } & \multicolumn{2}{|l|}{$24.257 \pm 24.354$} & \multicolumn{2}{|c|}{$44 \pm 24.98$} & 0.187 \\
\hline
\end{tabular}

Anti TNF-anti tumor necrosis factors

Table 8. Disease characteristics association between sarcopenia and non-sarcopenia group

\begin{tabular}{|c|c|c|c|c|}
\hline & & \multicolumn{2}{|c|}{ Result } & \multirow{3}{*}{ *P-value } \\
\hline & & No sarcopenia & Sarcopenia & \\
\hline & & \multicolumn{2}{|c|}{ Mean \pm SD / No. (\%) } & \\
\hline \multicolumn{2}{|c|}{ Duration from diagnosis (years) } & $6.311 \pm 4.719$ & $12.4 \pm 4.219$ & 0.008 \\
\hline \multicolumn{2}{|l|}{ BASDAI } & $4.104 \pm 1.77$ & $4.66 \pm 1.088$ & 0.497 \\
\hline \multirow{2}{*}{ Disease Activity } & Inactive & $18(40)$ & $1(20)$ & \multirow{2}{*}{0.382} \\
\hline & Active & $27(60)$ & $4(80)$ & \\
\hline
\end{tabular}

BASDAI, Bath Ankylosing Spondylitis Activity Index; SD, standard deviation; *t-test

Table 9. ROC analysis and validity of LM, SMI, handgrip, usual gait speed test to differentiate between patients

\begin{tabular}{|l|c|c|c|c|c|c|c|}
\hline & AUC & Cutoff & Sensitivity & Specificity & PPV & NPV & Accuracy \\
\hline LM (kg) & 0.710 & $\leq 46.984$ & 68 & 68 & 68 & 68 & $71 \%$ \\
\hline SMI & 0.537 & $>7.7$ & 68 & 50 & 57.6 & 61 & $53.7 \%$ \\
\hline Handgrip & 0.719 & $\leq 34$ & 56 & 80 & 73.7 & 64.5 & $71.9 \%$ \\
\hline Usual Gait Speed & 0.546 & $>0.92$ & 60 & 60 & 60 & 60 & $54.6 \%$ \\
\hline
\end{tabular}

LM, lean mass; SMI, skeletal muscle index; AUC, area under the curve

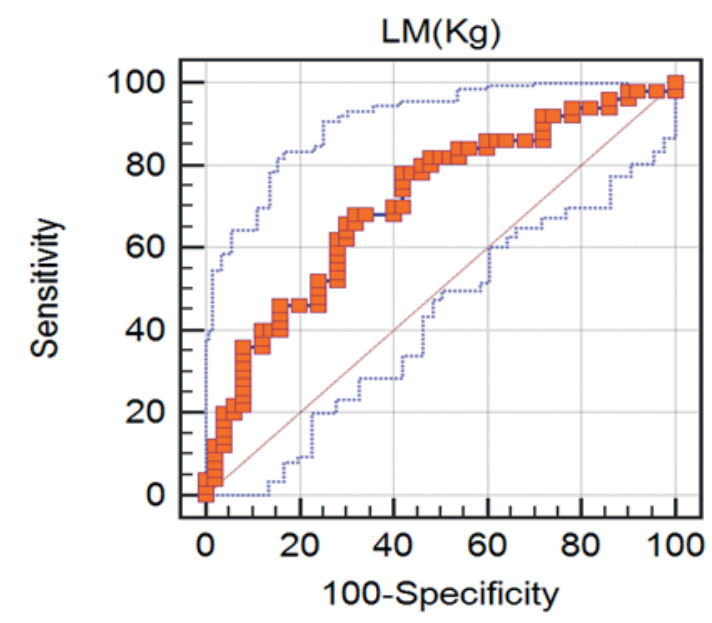

Fig. 3. ROC curve of LM of AS patients with sarcopenia 


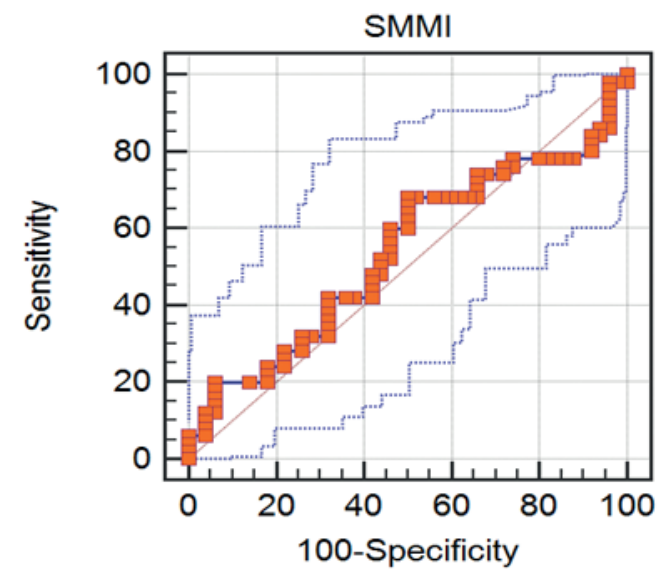

Fig. 4. ROC curve of SMI of AS patients with sarcopenia

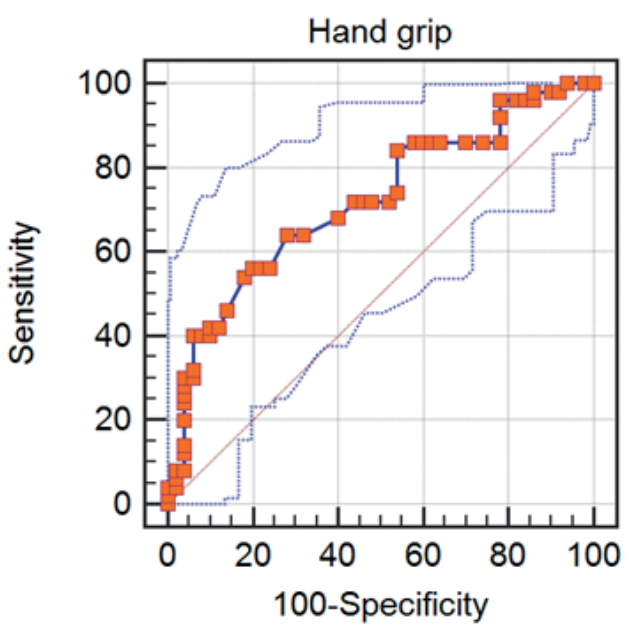

Fig. 5. ROC curve of handgrip of AS patients with sarcopenia

\section{Discussion}

To the best of our knowledge, this is the first study that assessed the prevalence of sarcopenia in a sample of Iraqi patients with Ankylosing Spondylitis.

Sarcopenia had been found in patients with younger age, lower BMI, longer disease duration, and lower physical activity. The prevalence of sarcopenia is not influenced by gender and smoking. The use of anti-TNF Lean mass and handgrip were fairly valid tests to differentiate between patients and controls.

This study showed the predominance of male with AS to female with a ratio of 15:1 which is comparable to another study conducted at Baghdad Teaching Hospital by Al-Osami et al. [20] and another one by Abdul-Wahid et al. [21], which showed the male to female ratio was $13: 1$ and $11: 1$, respectively.

In the current study, 10 of patients were found to have sarcopenia. These results are slightly lower than the results reported in a similar study at the
American College of Rheumatology/the Association of Rheumatology Health Professionals Annual Scientific Meeting in San Diego in 2017 [22], when the prevalence was $15 \%$ and $26 \%$ by applying Asian working group for sarcopenia (AWGS) criteria and Korea criteria respectively.

Barros et al. [23] and Barone et al. [24] showed the prevalence of sarcopenia equals $16.6 \%$ and $22.7 \%$, respectively.

The prevalence of presarcopenia in the current study was $6 \%$ in both groups which disagree with another two studies, EL Maghraoui et al. [25] and Barone et al. [24], where the prevalence of presarcopenia was much greater $49.3 \%, 36.3 \%$, respectively. These differences in the prevalence might be attributed to the following factors: sarcopenia is an age-related condition and the patients who enrolled in the former study were older compared to the mean age of patients in ours $(40.9 \pm 11$ years vs $36.64 \pm 7.684$ years). Another explanation is the differences in methodology and the cutoff values that had been used in the diagnosis of sarcopenia and pre-sarcopenia.

The results showed that the AS patients with sarcopenia had a lower mean value of BMI compared to patients without sarcopenia, but when the patients stratified according to their categories of BMI, the results demonstrated that sarcopenia was more common in normal and underweight patients $(80 \%$, and $20 \%$ ), respectively. These results are in agreement with the studies of Song et al. [22] and El Maghraoui et al. [25], where they revealed that AS patients with sarcopenia tend to have lower BMI than the patients without it.

Physical activity is the major stimulus for muscle anabolism and therefore may be of high importance in the prevention of muscle loss. Chronic diseases such as AS interfere with patients' physical functioning and quality of life. As a consequence, patients are at risk for physical inactivity and eventually loss of muscle mass [26, 27].

Data from our study revealed a statistically significant difference between the two groups (sarcopenia and non-sarcopenia groups) in that all of the patients with sarcopenia had reduced physical activity. These results are comparable to a study done in 2012 by Plasqui G. et al. [26], which assessed the relation of physical activity and body composition in AS patients and revealed that body composition is positively related to physical activity.

The data showed that the prevalence of sarcopenia was not associated with disease activity, while in other studies like Barone et al. [24] and 
El Maghraoui et al. [25], there was a correlation of BASDAI measurement with sarcopenia. BASDAI is exclusively based on subjective patient evaluation, which may be responsible for this conflict.

Further analysis showed that patients with sarcopenia had a statistically significant longer disease duration $(p=0.008)$, this in line with the other two studies Song et al. [22] and El Maghraoui et al. [25]. However, the results of the current study and these two studies were inconsistent with another two studies, Barone et al. [24] and Kim et al. [28], which revealed the disease duration does not influence the prevalence of sarcopenia.

Treatment with anti-TNF can induce a significant and sustained reduction in disease activity and systemic inflammation. This study estimates the effect of Anti-TNF on sarcopenia status as anti-TNF treatment is expected to prevent cachexia [25]. The data did not reveal significant differences between patients who were receiving treatment with anti-TNF and those who were not on treatment. The duration of treatment did not show a significant difference in the development of sarcopenia in AS patients. These results are consistent with two other studies Barone et al. [24] and El Maghraoui et al. [25]. The results and the other two mentioned studies are inconsistent with two other studies, Barros et al. [23], and Bariot et al. [29], which showed the reversal of sarcopenia status in patients who were treated with anti-TNF. These differences may be attributed to the availability of these medications and the class of Anti-TNF used in different studies.

However, it is the only study in Iraq that estimate the prevalence of sarcopenia in AS patients, which evaluate the preventable risk factors of sarcopenia in those group of population, for the purposes of the improvement of the quality of life.

\section{CONCLUSIONS}

Patients with AS are at higher risk of developing early sarcopenia. Lower BMI, longer disease duration increases the risk of sarcopenia, while gender differences, smoking, and the use of anti-TNF do not influence the risk of sarcopenia. Physical activity improves muscle strength and decreases the risk of sarcopenia.

\section{StUdY LIMITATIONS}

A small sample size of adult population was analyzed and the scope of the observation period is short $-1 / 2$ year. It is a single-center study, and the evaluation of disease activity was based on disease scoring, but not on biochemical indexes or pro-inflammatory cytokine measurements.

Conflict of interests: None

\section{References}

1. Braun J, Sieper J. Ankylosing spondylitis. Lancet. 2007;369 (9570):1379-90

2. Ljung L, Sundström B, Smeds J, et al. Patterns of comorbidity and disease characteristics among patients with ankylosing spondylitis-a cross-sectional study. Clin Rheumatol. 2018;37(3):647-53

3. Al-Bedri K. Prevalence, Clinical Features, and Radiological Features of Iraqi Patients with Ankylosing Spondylitis. J Nat Sci Res, 2014;24(4):53-9.

4. Ankylosing Spondylitis: Symptoms, Diagnosis, and Treatment. Johns Hopkins Arthritis Cent. [Internet] [cited 2019 Apr 24]; Available from: https://www.hopkinsarthritis.org/arthritis-info/ankylosing-spondylitis/

5. Sieper J, van derHeijde D, Landewe R, et al. New criteria for inflammatory back pain in patients with chronic back pain: a real patient exercise by experts from the Assessment of SpondyloArthritis international Society (ASAS). Ann Rheuma Dis, 2009;68(6):784-788.

6. Cruz-Jentoft AJ, Bahat G, Bauer J, et al. Sarcopenia: revised European consensus on definition and diagnosis. Age Ageing. 2019;48(1):16-31.

7. Muscaritoli M, Anker S, Argiles J, et al. Consensus definition of sarcopenia, cachexia, and pre-cachexia: joint document elaborated by Special Interest Groups (SIG) "cachexia-anorexia in chronic wasting diseases" and "nutrition in geriatrics." Clin Nutrition. 2010;29(2).

8. Ogawa, S, Yakabe M, Akishita M. Age-related sarcopenia and its pathophysiological bases. J Nat Sci Res, 2016;38(1):17.

9. Janssen I, Heymsfield SB, Ross R. Low Relative Skeletal Muscle Mass (Sarcopenia) in Older Persons Is Associated with Functional Impairment and Physical Disability. J Am Geriatr Soc. 2002;50(5):889-96.

10. Cruz-Jentoft A, Baeyens J, Bauer JM, et al. Sarcopenia: European consensus on definition and diagnosis. Age Aging. 2010; 39(4):412-23

11. Demirkapi M, Yildizgören MT, Güler $H$, Turhanoğlu AD. The Effect of Anti-Tumor Necrosis Factor-Alpha Treatment on Muscle Performance and Endurance in Patients With Ankylosing Spondylitis: A Prospective Follow-Up Study. Arch Rheumatol. 2017;32(4):309-14.

12. Gorial F, Mahmood Z, Al Obaidi S. Body Composition in Iraqi Women with Systemic Lupus Erythematosus. 2019;11(1). Available from: http://un.uobasrah.edu.iq/display_papers_ info.php?id $=6471$

13. Raychaudhuri SP, Deodhar A. The classification and diagnostic criteria of ankylosing spondylitis. J Autoimmun. 2014;48-49:128-33

14. World Health Organization. Obesity: Preventing and Managing the Global Epidemic. World Health Organization, 2000. 894(i-xii):1-253

15. The General Practice Physical Activity Questionnaire (GP$P A Q)$. In Irritable Bowel Syndrome in Adults: Diagnosis and Management of Irritable Bowel Syndrome in Primary Care 
[Internet]. Royal College of Nursing (UK); 2008 [cited 2019 Oct 16]. Available from: https://www.ncbi.nlm.nih.gov/books/ NBK51962/

16. Digital Smedly Hand Dynamometer - Hopkins Medical Products [Internet]. [cited 2019 Oct 15]. Available from: https:// www.hopkinsmedicalproducts.com/physical-therapy/p/Digital-Smedly-Hand-Dynamometer/

17. España-Romero V, Ortega FB, Vicente-Rodríguez G, et al. Elbow position affects handgrip strength in adolescents: validity and reliability of Jamar, DynEx, and TKK dynamometers. J Strength Cond Res. 2010;24(1):272-7.

18. Roberts H, Denison H, Martin H, et al. A review of the measurement of grip strength in clinical and epidemiological studies: towards a standardized approach. Age Ageing. 2011;40(4):423-9.

19. Working Group on Functional Outcome Measures for Clinical Trials. Functional outcomes for clinical trials in frail older persons: time to be moving. J Gerontol A Biol Sci Med Sci. 2008 Feb;63(2):160-4.

20. Al Osami MH, Hameed EK, Al Hamadani AM. Effect of HLA B27 Status and Body Mass Index on the Clinical Response to Infliximab in Ankylosing Spondylitis Patients. Indian Journal of Rheumatology. 2018; 13(1):2.

21. Abdul-Wahid K, Karhoot J, Al-Osami M, et al. Assessment of Serum Calprotectin (S-100 Protein) In Iraqi Patients with Ankylosing Spondylitis and Its Relation with Treatment and Disease Activity. IOSR Journal of Pharmacy and Biological Sciences (IOSR-JPBS) 2018;13(2):14-7

22. Song R, Lee SH, Choi JY, et al. Prevalence of and Factors Associated with Sarcopenia in Patients of Ankylosing Spondylitis. In ARTHRITIS \& RHEUMATOLOGY 2017; 1 (Vol. 69). 111 RIVER ST, HOBOKEN 07030-5774, NJ USA: WILEY.

23. Barros MA, Saad CS, Takayama L, et al. THU0266 Sarcopenia reversal in ankylosing spondylitis (AS) under anti-TNF therapy: A 24-month longitudinal analysis. Ann Rheum Dis. 2013;71(Suppl 3):244-5.

24. Barone M, Viggiani M, Anelli M, et al. Sarcopenia in Patients with Rheumatic Diseases: Prevalence and Associated Risk Factors. J Clin Med. 2018; 7(12):504.

25. El Maghraoui A, Ebo'o FB, Sadni S, et al. Is there a relation between pre-sarcopenia, sarcopenia, cachexia, and osteoporosis in patients with ankylosing spondylitis? BMC Musculoskelet Disord. 2016;17(1):268.

26. Plasqui $G$, Boonen a, Geusens $P$, et al. Physical activity and body composition in patients with ankylosing spondylitis. Arthritis Care Res. 2012;64(1):101-7.

27. Jasim W, El-Yassin. H, Abdulatif $\mathrm{N}$. Interventional biological markers for sarcopenia and muscle frailty in Iraqi subjects. Iraq Med J. 2017; 1(2):29-36.

28. Kim SC, Lee YG, Park S-B, etal. Muscle Mass, Strength, Mobility, Quality of Life, and Disease Severity in Ankylosing Spondylitis Patients: A Preliminary Study. Ann Rehabil Med. 2017;41(6):990-7.

29. Briot, K, Gossec L, Kolta S, et al. Prospective assessment of body weight, body composition, and bone density changes in patients with spondyloarthropathy receiving anti-tumor necrosis factor-alpha treatment. J. Rheumatol. 2008;35(5):855-61.

Submitted: 17.06 .2021

$\triangle$ Correspondence address:

Younis MM, Baghdad

10047

Iraq

Baghdad, 10047, 7733962400

m31947683@gmail.com 